\title{
White Beam Topography of 300 mm Si - Wafers
}

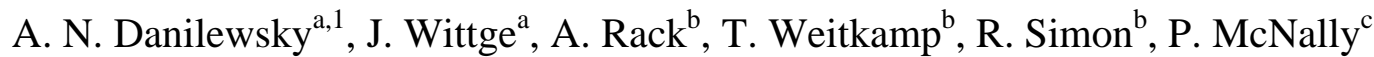

${ }^{a}$ Kristallographisches Institut, University Freiburg, Freiburg, Germany

bInstitut für Synchrotronstrahlung, Research Centre Karlsruhe, Karlsruhe, Germany

${ }^{\mathrm{c}}$ Research Institute for Networks and Communications Engineering, Dublin City University,

Dublin, Ireland

\section{${ }^{1}$ Corresponding Author:}

\author{
A. N. Danilewsky \\ Kristallographisches Institut \\ Hermann-Herder-Str. 5 \\ D-79104 Freiburg i. Br. \\ Germany \\ phone: +49-761-203-6450 \\ fax: +49-761-203-6434 \\ E-Mail: a.danilewsky@krist.uni-freiburg.de
}




\begin{abstract}
Synchrotron X-ray topography is well suited for a detailed characterisation of the real structure of single crystals and devices based on single crystalline materials. The nature and distribution of dislocations, stacking faults, inclusions etc. as well as long range strain from processing are of high interest especially in semiconductor wafers and electronic devices. To overcome the limitations of the classical photographic film method, we use a high resolution digital imaging detector. The digital scan of selected reflections allows the fast mapping of large sample areas with high resolution in combination with high dynamic range of the CCDcamera. We report our first applications on the metrology of $300 \mathrm{~mm}$ Si wafers.
\end{abstract}




\section{Introduction}

Advanced Si device manufacturing is characterized by the increasing size of the Si-wafers, the size and integration of device structures and the number of process steps resulting in increasing strain, defects and - worst case - micro-cracks and fracture [1]. Yield and reliability of microelectronic and micromechanic devices based on single crystals depend strongly from the control and prevention of defect generation [2].

In general X-ray topography is non-destructive imaging technique to analyse this kind of defects. Especially the white beam synchrotron topography (SXRT) making use of synchrotron radiation is well suited for the characterisation of long and short range strain in single crystals (e.g. dopant striations, dislocations) as well as in devices [3].

It is based on recording a Laue-pattern of reflections where each reflection contains a topograph from the same investigated crystal position. Typically the patterns are collected on conventional X-ray films. Each topograph corresponds to a different diffraction vector and is magnified, photographed and digitalised with a conventional light microscope. Replacing and developing the films as well as digitalising the topograph is very time consuming which can be overcome by using a digital X-ray imaging detector [4]. The limited area of such a X-ray camera allows to record only one reflection at a time. But for the characterization of defects in semiconductor single crystals and devices respectively, generally one single reflection, e.g. 220, is sufficient because the types of dislocations are well known. Si crystallizes in the diamond-structure, where the preferential dislocations are well known and described with the aid of the Thompson tetrahedron [5].

The Topo-Tomo beamline at the synchrotron light source ANKA at the research centre Karlsruhe (Forschungszentrum Karlsruhe) is now upgraded for a fast, non-destructive metrology of wafers up to $300 \mathrm{~mm}$. 


\section{Experimental Details}

The synchrotron light source ANKA at the research centre Karlsruhe (Forschungszentrum Karlsruhe) is operated with a ring electron energy of $2.5 \mathrm{GeV}$ and beam currents of $180-80$ mA. It results in the characteristic wavelength of $2 \AA$ which is perfectly suited for topography. Consequently, a dedicated experimental set up for synchrotron white beam X-ray topography is installed at the beamline Topo-Tomo. For high resolution white beam topography the beamline is ran without any optical components between source point and experiment. Only one Be window is placed at the end of the vacuum tube. Here, directly in front of the exit window, a vacuum slit system allows to change from large area (up to $10 \mathrm{x} 10 \mathrm{~mm}^{2}$ ) to section topography (15 $\mu \mathrm{m}$ x $10 \mathrm{~mm}$ ) very easily. The details about the Topo-Tomo beamline and the experimental station at ANKA are given in refs. [6,7].

Fig. 1 shows the actual experimental station of the Topo-Tomo beamline, where topography (diffraction imaging) as well as tomography (radiography) can be performed. The beamline is upgraded to handle $300 \mathrm{~mm}$ or even larger wafers. Any position on the wafer can be adjusted by two high precision linear drives (Micos $\mathrm{GmbH}, 300 \mathrm{~mm}$ travel range). The standard photographic films in use are Agfa D3sc (single coated, grain size of $0.3 \mu \mathrm{m}$ ) for adjustment and for high resolution exposures Slavich VRP-M (grain size $0.05 \mu \mathrm{m}$ ). In addition there is a digital X-ray camera [4,8,9] available to image single topographs with excellent spatial resolution and increased dynamic range. 


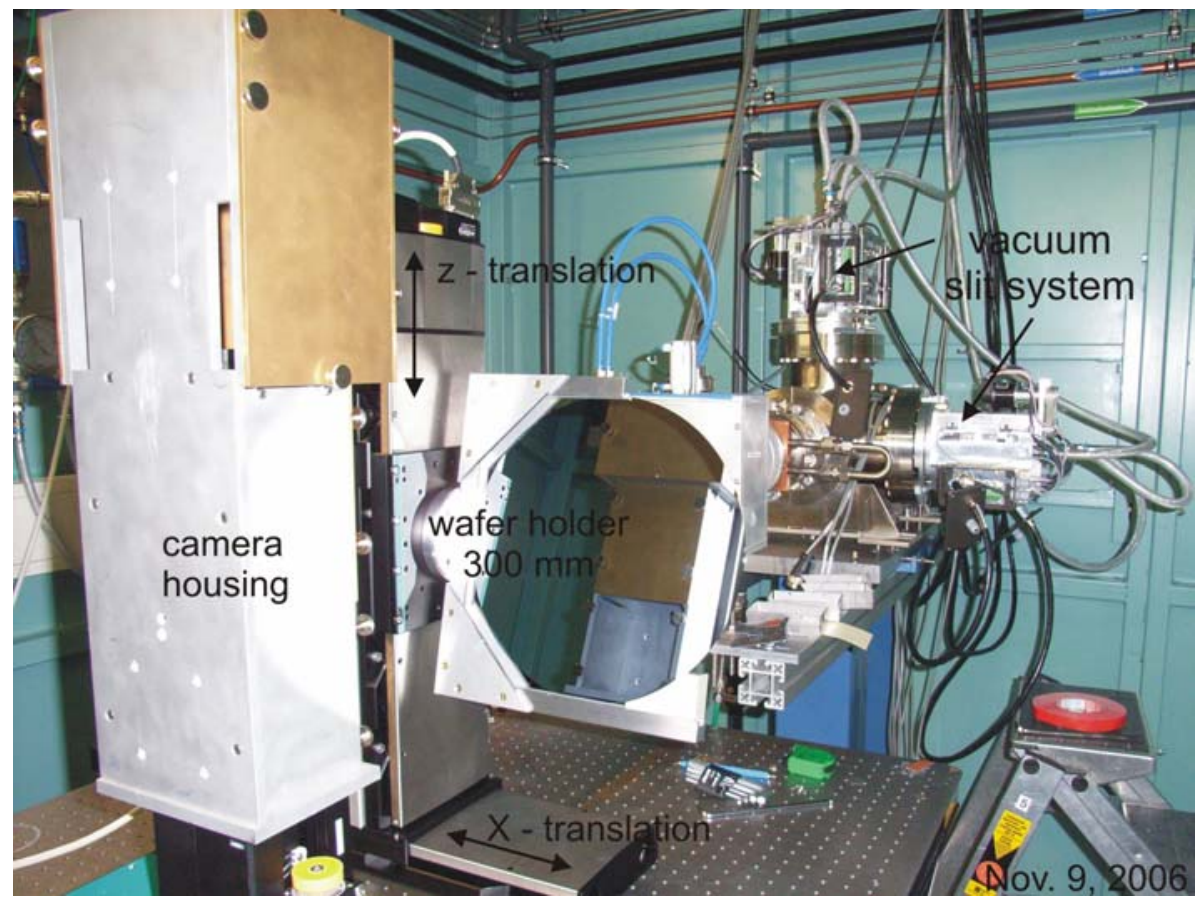

Fig. 1: Experimental station of the digital white beam topography with commercial $300 \mathrm{~mm}$ Si wafer (tilted by $12^{\circ}$ to adjust the 220 -reflection)

The digital imaging system consists of a $\mathrm{CdWO}_{4}$ scintillating single-crystal of $40 \mu \mathrm{m}$ thickness, polished on both sides and glued to a YAG substrate. Its luminescence image is projected and magnified onto a CCD camera (PCO4000, 11 MPixel, 14bit ADC, 5.000 graylevels) via a microscope optic. Using this system, the achieved lateral resolution is approximately $5 \mu \mathrm{m}$ (2.5 $\mu \mathrm{m}$ pixel size). To reduce noise the CCD chip is cooled. Additionally, a dark field correction is done for each recorded topograph.

Whereas the large sized X-ay film with $13 \mathrm{x} 18 \mathrm{~cm}^{2}$ collects a high number of topographs during one exposure, the camera with an active area of only 25 x $25 \mathrm{~mm}^{2}$ has to be adjusted carefully for one single reflection. For that the camera housing is mounted on linear drives to get the selected reflections. In case of Si the most important reflections are 220 or 004 . A 
standard (001)-wafer has to be tilted by $12^{\circ} / 9.75^{\circ}$ at a distance of $9 / 19 \mathrm{~cm}$ from wafer to film/detector.

This allows the fast monitoring of a high number of positions on large wafers without loosing the waiting time during the developing procedure of the photographic films. Actually the digital imaging is available for large area and section transmission topography.

For demonstration a commercial $300 \mathrm{~mm} \mathrm{Si} \mathrm{wafer} \mathrm{with} \mathrm{a} \mathrm{thickness} \mathrm{of} \mathrm{about} 450 \mu \mathrm{m}$ was used, which shows a low number of defects with a range of contrasts.

\section{Results and discussion}

The topographs collected on high resolution film and by digital camera show a similar high resolution [10]. Fig. 2 compares 220 section topographs from nearly perfect Si taken by film and camera respectively. The sharp Pendellösung fringes indicate the high perfection of the wafer at this position. If needed, a mapping with an accuracy of $1 \mu \mathrm{m}$ becomes possible. The computer control allows the automated long term mapping of large selected wafer areas. To locate defects, such a mapping can be performed with drastically shortened exposure times of some tenths of seconds. Once the defects are found, the topography is repeated with longer exposure time for better signal to noise ratio. Details become visible at about 4-5 minutes for the section mode and at about 20 minutes the same quality as for high resolution film is realized. The exposure time needed for the optimum quality of section topography is $10-30 \%$ shorter than for high resolution film and may be reduced again by optimising the software for post image processing. 


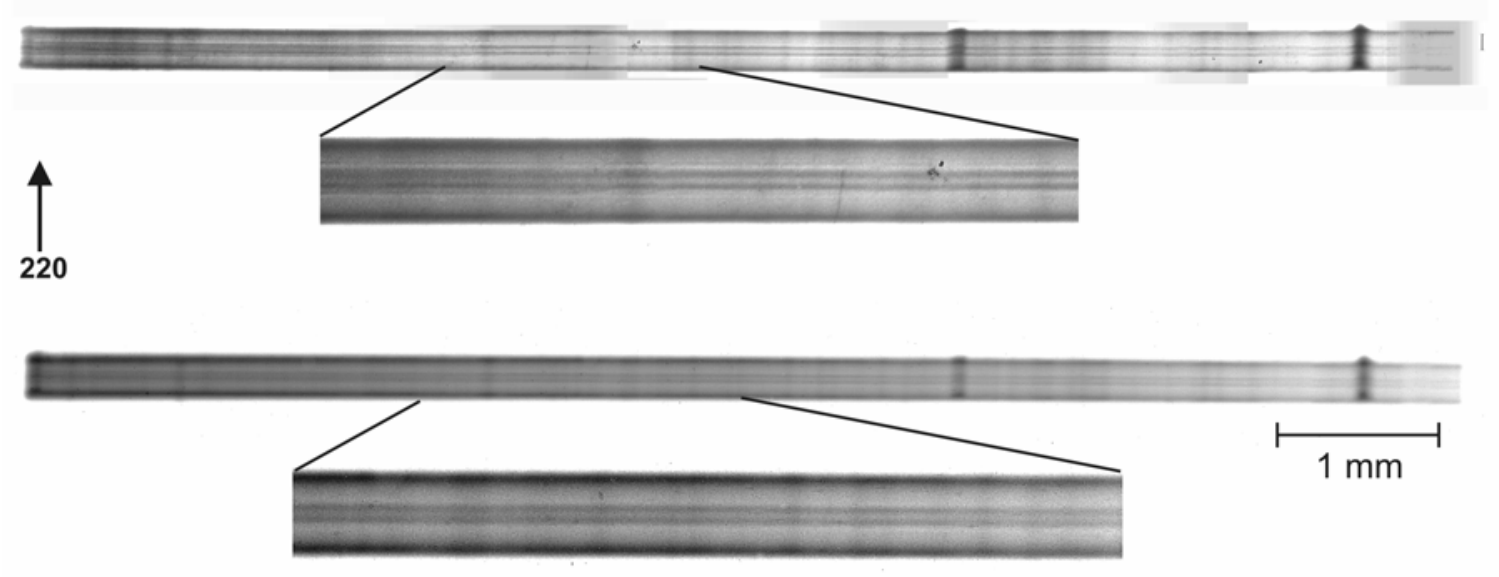

(a)

Fig. 2: Pendellösung fringes in perfect Si, 220 - reflection

(a) High resolution film, distance $9 \mathrm{~cm}, 31 \mathrm{~min}$ (SXRT20, image processed)

(b) High resolution camera, distance $19 \mathrm{~cm}, 20 \mathrm{~min}$ (dark corrected, image processed)

A series of topographs was taken in the large area mode at the same wafer position but varying the exposure time from 1 to 40 minutes to find the lower limit for the exposure time. In case of high resolution film the exposure time is $4-5$ minutes, depending from the actual values of the beam current. In fig. 3 two topographs are compared which are taken with camera for 1 and 5 minutes respectively. The large contrasts near the edge and the notch of the wafer are well visible, still at short exposure time. Curved dislocation lines can be identified as well as large black contrasts corresponding to micro-cracks. At shorter exposure time the outshine of strong contrasts is reduced and more details of the dislocations and micro-cracks become visible.

The very long exposure time of 40 minutes reduces the back ground noise but gives no additional information. In contrary the high strain areas become overexposed and therefore the details are less visible. The best outcome is achieved with the 1 minute exposure, despite the back ground noise. 


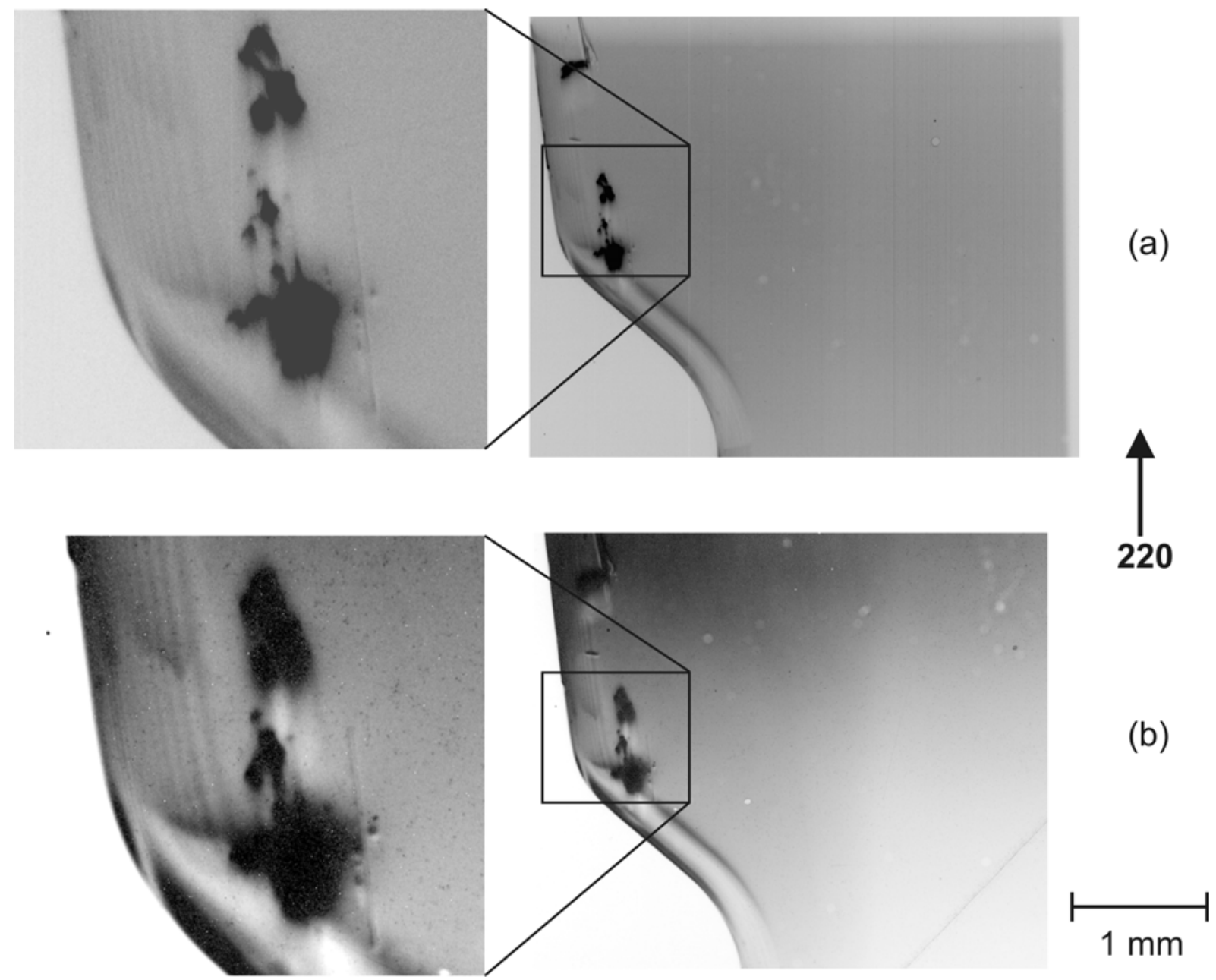

Fig. 3: Large area topography near the notch of a Si - wafer with defects, taken with camera (dark corrected and image processed):
(a) 1 minute exposure time
(b) 5 minutes exposure time (same as for high resolution film) 


\section{Conclusions and outlook}

Digital synchrotron X-ray - topography is well suited for the rapid metrology of large Si wafers in section and large area transmission mode. The exposure time can be reduced drastically without loosing information. Pendellösung fringes as well as dislocations, microcracks and long range strain become visible by choosing one appropriate diffraction vector. For the detailed characterisation of dislocations and the Burgers-vector analysis additionally exposures with an adequate choice of diffraction vectors are needed.

In future the resolution will be increased using camera optics with higher magnification. The improvement for back reflection mode is under construction.

\section{Acknowledgements}

xyz

\section{References}

[1] S. M. HU, J.Appl. Phys. 70(6) (1991), R53-R80.

[2] R. F. COOK, J. Mat. Sci. 41 (2006), 841-872.

[3] T. TUOMI, K. NAUKKARINEN, P. RABE, phys. stat. sol.(a) 25 (1974), 93.

[4] WEIDEMANN G., GOEBBELS J., WOLK TH., RIESEMEIER, H., BESSY Annual Report 2001, 249-250.

[5] N. THOMPSON. Proc. Phys. Soc. 66B (1953) 481.

[6] A. N. DANILEWSKY, R. SIMON, A. FAULER, M. FIEDERLE AND K. W. BENZ, Nuclear Instruments and Methods in Physics B 199(1) (2003), 7174.

[7] R. SIMON AND A. N. DANILEWSKY, Nuclear Instruments and Methods in Physics B 199(1) (2003), 550-553.

[8] U. BONSE and F. BUSCH, Prog. Biophys. Molec. Biol. 65 (1996), 133-169.

[9] A. N. DANILEWSKY, J. WITTGE, A. RACK, T. WEITKAMP, R. SIMON, NIM-B 2007 submitted

[10] A. N. DANILEWSKY, J. WITTGE, A. RACK, T. WEITKAMP, R. SIMON, Z. Krist. Suppl. 25 (2007). 